\title{
SURGICAL TREATMENT ALTERNATIVE TO THE HIP DISARTICULATION IN PACIENTS WITH MALIGNANT TUMOR OF THE PROXIMAL FEMUR
}

doi: $10.2478 /$ rojost-2018-0045

\author{
M. Moga ${ }^{1,2}$, G. Dinache ${ }^{1}$, M. Pogărășteanu ${ }^{1}$, S. Lupascu ${ }^{1}$ \\ "Dr. Carol Davila" Universitary Emergency Central Military Hospital, Bucharest, Romania \\ The Orthopaedics - Traumathology Clinic \\ 2 "Carol Davila" University of Medicine and Pharmacy, Bucharest, Romania
}

Background. Primary bone tumors are rare and affect especially young persons, being at the same time very aggressive and mutilating, often also implicating the disarticulation of the affected hip. That is why it is necessary to find alternatives of treatment, so that these young people to be able to keep their normal functionality and mental comfort.

Objectives. The objective of this paper was to be able to offer a surgical treatment alternative to the hip disarticulation in case of malignant tumors of the proximal femur in young patients.

Methods. We present the case of a 25 -year-old female patient, who was hospitalized in our clinic for pain and functional impairment of the right hip. The clinical and radiological exams established the diagnosis of fracture of pathological bone of the proximal femur and it raised the suspicion of a tumor with malignant characters. We performed arteriography of the right inferior member, which showed a very good vascularized tumor, possibly vascular, localized to the proximal femur, arterially powered by the branches of right profunda femoris artery. We surgically intervened and made an incisional biopsy with anatomopathological exam, which established the diagnosis of osteosarcoma. Then, we practiced the segmental resection of the tumor, preceded by the embolization of the right femoral artery and after that the reconstruction with bipolar cemented modular prosthesis of the right hip. The patient had a favorable evolution, she did neuromotor rehabilitation, and then she presented to the oncologist for the initiation of adjuvant chemotherapy, which she followed conformably to the recommendations.

Keywords: osteolytic tumor of the proximal femur, reconstruction prosthesis, hip disarticulation 